Voix et Images

\title{
MÉMOIRE DU CONTE ET RENOUVELLEMENT DU ROMAN QUÉBÉCOIS CONTEMPORAIN
}

\section{MARISE BELLETÊTE et MARIE-PASCALE HUGLO}

Volume 43, numéro 3 (129), printemps-été 2018

Mémoire du conte et renouvellement du roman québécois

contemporain

URI : https://id.erudit.org/iderudit/1051082ar

DOI : https://doi.org/10.7202/1051082ar

Aller au sommaire du numéro

Éditeur(s)

Université du Québec à Montréal

ISSN

0318-9201 (imprimé)

1705-933X (numérique)

Découvrir la revue

Citer ce document

BELLETÊTE, M. \& HUGLO, M.-P. (2018). MÉMOIRE DU CONTE ET

RENOUVELLEMENT DU ROMAN QUÉBÉCOIS CONTEMPORAIN. Voix et Images,

43(3), 7-11. https://doi.org/10.7202/1051082ar d'utilisation que vous pouvez consulter en ligne.

https://apropos.erudit.org/fr/usagers/politique-dutilisation/ 


\title{
MÉMOIRE DU CONTE ET RENOUVELLEMENT DU ROMAN QUÉBÉCOIS CONTEMPORAIN
}

\author{
$++$ \\ MARISE BELLETÊTE \\ Université du Québec à Rimouski \\ MARIE-PASCALE HUGLO \\ Université de Montréal
}

Le conte bénéficie, dans la culture contemporaine, d'un regain de popularité notable. Associé, en premier lieu, à l'oralité et à la performativité, le conte québécois s'est renouvelé en milieu numérique et urbain. Traditionnellement «transmis oralement de génération en génération dans une société donnée dont il est en quelque sorte l'imaginaire collectif et parfois la mémoire ${ }^{1} "$, comme le définissait Jeanne Demers, ce genre bref et merveilleux se situant «en marge du réel ${ }^{2}$ » s'est ouvert sur le monde et le présent. Le succès de Fred Pellerin et les nombreux regroupements et festivals de conteurs (le Rendez-vous des Grandes Gueules, le Festival interculturel du conte du Québec, etc.) fleurissant un peu partout dans la province en sont des exemples probants, faisant la part belle au renouveau de la parole conteuse.

La résurgence contemporaine du conte aurait ainsi à voir avec sa capacité de relancer des archétypes et des imaginaires communs sous des formes et des modes très actuels. On le retrouve au théâtre (prenons pour exemple la pièce La Belle et la Bête [2011], une création de Michel Lemieux et de Victor Pilon au Theâtre du Nouveau Monde, ou encore les pièces d'Elfriede Jelinek, tirées de Drames de princesses [2006], mises en scène à l'Espace Go par Martin Faucher en 2011); au cinéma (le film L'Odyssée d'Alice Tremblay [2002], réalisé par Denise Filiatrault); en danse (dans le ballet Cendrillon. "Celle qui, dit-on, aurait perdu sa chaussure», créé par Stijn Celis ${ }^{3}$ pour les Grands Ballets canadiens de Montréal en 2003, et dans celui de La Belle au bois dormant [2009] de Mats $\mathrm{Ek}^{4}$, par exemple); de même que dans le

1 Jeanne Demers, Le conte. Du mythe à la légende urbaine, Montréal, Québec Amérique, coll. «En question», 2005, p. 30.

2 Jean-Marc Massie, Petit manifeste à l'usage du conteur contemporain. Le renouveau du conte au Québec, Montréal, Planète rebelle, 2001, p. 22. Massie donne du conte la définition suivante: "Au sens strict du terme, le conte est une fiction balisée par des formules d'ouverture et de fermeture [...] dont le lieu et le temps sont indéterminés, et où le héros est connu de manière très générale [...]. Le temps et l'espace du conte sont respectivement hors du temps et de l'espace réels. Il s'agit souvent d'un récit court et merveilleux d'aventures imaginaires, suivant une logique simple.» Ibid., p. 20.

3 Voir: http://www.grandsballets.com/fr/a_propos/choregraphes/stijn-celis (page consultée le 5 avril 2018).

4 Voir: https://grandsballets.com/fr/spectacles/detail/belle-au-bois-dormant-la-2 (page consultée le 10 juin 2018). 
milieu des arts visuels et de la photographie (pensons à Catherine Rondeau et à son exposition «De l'autre côté du miroir ${ }^{5}$ ", ainsi qu'à la collection «Fallen Princesses ${ }^{6}$ » de Dina Goldstein, présentée au Musée de la Femme de Longueuil en 201377. Mais la vitalité du conte dans la culture québécoise concerne aussi la littérature. C'est précisément sa résurgence dans le roman québécois contemporain qui nous occupe dans ce dossier. La lecture attentive d'œuvres pour la plupart peu étudiées vise à donner un aperçu critique de la prégnance du conte dans la littérature contemporaine tout en faisant converger les modalités et les enjeux de ses réécritures.

Il s'agit moins, dans ce domaine, d'écrire de nouveaux contes que de renouveler l'écriture avec le conte: le conte traverse nombre de romans et récits contemporains sur les plans de l'intertexte, de la langue et de la trame narrative, textes tissés d'une mémoire largement partagée, mais qui ne souscrivent pas pour autant aux attentes du genre, voire les dévoient. Il semble en effet que la mémoire du conte, attachée à l'enfance et à l'imaginaire, constitue un terrain d'invention littéraire: elle renouvelle l'horizon du récit en jouant de la friction entre les attentes réalistes d'ordre romanesque et la persistance du merveilleux et des monstres attachés à l'univers des contes.

Loin de reléguer le conte à un passé idéalisé et révolu, sa reprise dans les œuvres littéraires contemporaines lui confère une actualité saillante: les éléments structurants des contes y sont mobilisés, engagés dans un mouvement de transformation aussi bien formelle que narrative, thématique et langagière, renouvelant et redynamisant le récit à partir de motifs et de modèles culturels puissants. Les lectures critiques réunies dans ce dossier, empruntant des pistes d'analyse multiples (psychanalytique, féministe, comparatiste, intertextuelle, ethnographique), cherchent ainsi à prendre la mesure de la présence du conte dans le récit québécois des dernières décennies. Se dégage de l'ensemble des œuvres abordées dans ce dossier une polarisation entre l'inactuel et le contemporain. D'une part, le roman s'inscrit, à la manière des contes, dans un espace-temps éloigné ou impossible à situer, renouant avec des archétypes ancrés dans nos imaginaires subjectifs et collectifs. Les mondes de David Clerson, de Guillaume Corbeil, d'Audrée Wilhelmy ou encore d'Anne Hébert mettent ainsi à distance nos repères pour convoquer, chacun à sa manière, une dimension non réductible au connu, au proche. D'autre part, les figures et les motifs issus des contes deviennent des modèles à l'aune desquels les préoccupations contemporaines sont (ré)évaluées. Les romans de Simon Boulerice et de Clara B.-Turcotte font ainsi émerger des situations ancrées dans le Québec du Xxi ${ }^{e}$ siècle, s'adossant au conte dans un geste subversif. Dans tous les cas, les contes et leurs variantes permettent de représenter, avec une efficacité empreinte d'oralité, des mondes où la violence et la transgression se déploient avec force. Car si la merveille miroite parfois encore, le côté «noir», cruel et terrifiant surtout domine, les contes donnant accès, sans détour, à une part d'ombre refoulée que les romans explorent tout en se détournant d'une finalité morale comme d'une

5 Voir: https://catherinerondeau.com/portfolio-autre-cote-miroir (page consultée le 10 juin 2018).

6 Voir: https://www.dinagoldstein.com/fallen-princesses (page consultée le 10 juin 2018).

7 Voir: http://grandquebec.com/archives-du-grandquebec/cOntes-defaits (page consultée le 10 juin 2018). 
résolution heureuse, non sans renverser au passage les codes attendus du genre féminin (dont la princesse est l'archétype) ou de l'amour. Les personnages d'adolescents et de jeunes adultes, très présents dans le corpus, incarnent bien la portée initiatique des récits qui «bricolent» avec le conte - initiation que l'on voit rejouée, dramatisée mais aussi suspendue, comme si le passage à l'âge adulte ne se trouvait partiellement reconduit que pour être mis en question, perverti ou se heurter à un inaboutissement problématique.

En ouverture de ce dossier, la lecture du roman Les fous de Bassan nous rappelle que les résurgences contemporaines du conte se profilent, pour ainsi dire, «à l'ombre d'Anne Hébert». L'article de Sylvie Vignes propose en ce sens une (re)lecture des diverses voix narratives prenant souffle à l'«ombre des contes»: "placé sous le signe du merveilleux avec le toponyme de Griffin Creek ${ }^{8}$ ", mais aussi marqué par la violence des meurtres qu'il relate, le roman entretient, par sa tonalité onirique et sa langue singulière, un rapport significatif avec l'univers atemporel et l'«inquiétante étrangeté» du conte. Vignes y repère la forte présence des êtres hybrides, celle de la sirène notamment. À partir des liens entre le «mythe antique des sirènes ornithomorphes » et le conte moderne La petite sirène de Hans Christian Andersen, elle interroge la multiplicité de cette figure qui hante le roman, " où la femme des eaux est tour à tour prédateur et proie, appât et victime sacrificielle ${ }^{9}$ ». En soulignant les ressemblances entre les contenus du rêve et celui du conte, l'article invite, dans une perspective psychanalytique, à considérer la «réversibilité» et le brouillage des «frontières entre le bien et le mal ${ }^{10}$ » dans l'œuvre hébertienne.

La noirceur des contes semble bel et bien retenir l'attention des créateurs, surtout lorsqu'il est question de mettre en scène des rapports homme-femme. Violence, cruauté, transgression sexuelle et enfermement reviennent effectivement beaucoup dans les œuvres littéraires renouant avec ce genre. On en retrouve les traces dans le roman Les sangs d'Audrée Wilhelmy, qui dépeint les sept femmes d'un ogre rappelant Barbe-Bleue non pas comme des victimes, mais comme des femmes recherchant à assouvir leurs fantasmes, souvent sadomasochistes, et leur pulsion de mort. Adoptant une perspective féministe, Marie-Hélène Larochelle, dans «Ras-le-bol du care», s'intéresse donc à la "dialectique constante entre le care et la violence (symbolique ou physique; métaphorique ou réelle), qui trouve ses racines dans les archétypes des contes de fées ${ }^{11}$ ", à travers sa lecture des Sangs d'Audrée Wilhelmy et de Demoiselles-cactus de Clara B.-Turcotte. Larochelle montre en quoi, dans ces deux romans, la posture du féminin se dégage de toutes les attentes d'une éthique du soin. Les contes peuvent dès lors être considérés comme un «héritage contaminé ${ }^{12}$ » que l'on doit faire éclater.

8 Voir, dans le présent dossier, Sylvie Vignes, «L'ombre des contes dans Les fous de Bassan d'Anne Hébert», p. 16.

9 Ibid., p. 24.

10 Ibid., p. 26.

11 Voir, dans le présent dossier, Marie-Hélène Larochelle, «Ras-le-bol du care. Une appropriation des codes du conte», p. 32.

12 Ibid., p. 40. 
Si certains personnages féminins rejettent avec force les rôles traditionnels et les princesses du passé, d'autres en sont si captivés qu'ils cherchent à les réinventer, même si, pour ce faire, ils doivent les détourner vers un éloge de la méchanceté. C'est le cas dans Javotte de Simon Boulerice, un antépisode de Cendrillon se déroulant au xxi siècle: la narratrice, fantasmant sur le modèle de la princesse bafouée puis reconnue, se plaît à concocter des scénarios de vengeance variés et délirants. Marise Belletête s'intéresse à l'actualisation subversive et parodique que le roman propose à notre époque, alors que $l^{\prime}$ « [o]n n'a plus les contes que $l^{\prime}$ on avait ${ }^{13}$ ". Elle montre à quel point la mémoire accumulée et éclatée des contes participe à l'ambiguité identitaire de la narratrice. Prisonnière de ces images de princesses et de l'édulcoration disneyenne, elle cherche simultanément à s'y comparer et à les mettre à mal.

Les récits recueillis dans Trois princesses de Guillaume Corbeil réinventent, pour leur part, Blanche-Neige, Cendrillon et La Belle au bois dormant. Corbeil propose aussi des réécritures qui démontent et reconstruisent les vies de ces héroïnes dans un effort similaire de dénonciation contre le règne des apparences, en respectant toutefois avec fidélité les schémas narratifs originaux des contes sans les détourner. En suivant le fil intertextuel de la réécriture au gré des variantes des mythes et des contes, Carmélie Jacob met en relief les figures de l'héroïne persécutée et de la «mèresécutrice» pour mieux dégager les questions de la filiation et du double qui donnent leur unité aux trois récits de Corbeil. En ressort «une réflexion sur la relation de la femme à son image ${ }^{14}$ » étrangement actuelle.

Parfois, ce sont aussi les échos du merveilleux et de l'étrange qui rappellent le conte, dans des mondes déroutants comme celui que nous présente David Clerson avec Frères, où un garçon parti à l'aventure devient un enfant-chien. Sophie Ménard aborde également la violence et les désordres de la filiation dans le roman, tout en débusquant les "motifs "contiques" » et les "scripts rituels qui régissent les manières de faire les jeunes garçons dans nos sociétés occidentales ${ }^{15}$ ", ajoutant ainsi une dimension «masculine» au dossier, si l'on peut dire, puisque ce sont surtout des personnages féminins qui retiennent l'attention dans les autres contributions.

La réécriture romanesque des contes est ainsi abordée sous divers angles. Depuis l'«inquiétante étrangeté» jusqu'à la reprise des figures de princesses, depuis le questionnement de la filiation jusqu'à la dénonciation des visées moralisantes désuètes et des idéologies dépassées, c'est, chaque fois, la capacité de remodelage à l'infini des contes qui s'impose dans les analyses ici rassemblées, le conte devenant un espace de variation ${ }^{16}$, «un schéma dynamique susceptible de reproductions

13 Simon Boulerice, Javotte, Montréal, Leméac, 2012, p. 105.

14 Voir, dans le présent dossier, Carmélie Jacob, «La reine est morte. Reflets de la filiation dans Trois princesses de Guillaume Corbeil», p. 72.

15 Voir, dans le présent dossier, Sophie Ménard, «Trésors du roman et du conte. Frères de David Clerson», p. 75.

16 Pour Claude Bremond, la structure même du conte est constituée de "pièces» permutables qui peuvent être reprises et échangées d'un conte à l'autre, se (ré)agençant sans cesse pour construire de nouvelles variantes du conte ou de nouveaux récits: «Le conte se présente comme le jeu de Meccano dans la caisse de jouets d'un enfant. Il y a des thèmes, pièces fixes, plus ou moins désassemblées, à partir desquelles le conteur, comme l'enfant, bricole une nouvelle construction.» Claude Bremond, «Le Meccano du conte», Le magazine littéraire, no 150, juillet-août 1979, p. 13-16. 
personnelles plus ou moins fidèles ${ }^{17}$ ». Pourtant - et c'est là une des dimensions qui ressort des articles du dossier -, tous les romans étudiés touchent à quelque chose d'interdit qui ne se pourrait dire autrement que dans une langue inédite (mais investie de mémoire), dans la reprise de motifs archaïques et d'imaginaires inactuels, dans une tension entre réalisme et merveilleux, enchantement et cynisme.

17 Jean Bellemin-Noël, Les contes et leurs fantasmes, édition revue, corrigée et augmentée, Montréal, Éditions Balzac, coll. «L'écriture indocile», 1995, p. 13. 